\title{
DÜBLIN
}

Technological University Dublin ARROW@TU Dublin

\section{A Note on Hopfian and Co-Hopfian Abelian Groups}

\author{
Brendan Goldsmith \\ Technological University Dublin, brendan.goldsmith@tudublin.ie \\ Ketao Gong \\ Technological University Dublin, ketao.gong@mydit.ie
}

Follow this and additional works at: https://arrow.tudublin.ie/scschmatcon

Part of the Mathematics Commons

\section{Recommended Citation \\ This Conference Paper is brought to you for free and open access by the School of Mathematics at ARROW@TU Dublin. It has been accepted for inclusion in Conference papers by an authorized administrator of ARROW@TU Dublin. For more information, please contact arrow.admin@tudublin.ie, aisling.coyne@tudublin.ie, gerard.connolly@tudublin.ie.}

Goldsmith, B. \& Gong, K. (2012). A Note on Hopfian and Co-Hopfian Abelian Groups. Proceedings of the American Mathematical Society, vol. 576, pg. 129-136. doi:10.1090/conm/576/11356. 


\title{
School of Mathematics \\ Articles
}

Dublin Institute of Technology

Year 2012

\section{A Note on Hopfian and co-Hopfian Abelian Groups}

\author{
Brendan Goldsmith* Ketao Gong ${ }^{\dagger}$
}

\footnotetext{
*Dublin Institute of Technology, brendan.goldsmith@dit.ie †DIT, ketao.gong@mydit.ie

This paper is posted at ARROW@DIT.

http://arrow.dit.ie/scschmatart/1
} 


\section{— Use Licence}

\section{Attribution-NonCommercial-ShareAlike 1.0}

You are free:

- to copy, distribute, display, and perform the work

- to make derivative works

Under the following conditions:

- Attribution.

You must give the original author credit.

- Non-Commercial.

You may not use this work for commercial purposes.

- Share Alike.

If you alter, transform, or build upon this work, you may distribute the resulting work only under a license identical to this one.

For any reuse or distribution, you must make clear to others the license terms of this work. Any of these conditions can be waived if you get permission from the author.

Your fair use and other rights are in no way affected by the above.

This work is licensed under the Creative Commons Attribution-NonCommercialShareAlike License. To view a copy of this license, visit:

- URL (human-readable summary):

http://creativecommons.org/licenses/by-nc-sa/1.0/

- URL (legal code):

http://creativecommons.org/worldwide/uk/translated-license 


\title{
A Note on Hopfian and co-Hopfian Abelian Groups*
}

\author{
B. Goldsmith \\ School of Mathematical Sciences \\ Dublin Institute of Technology \\ Aungier Street, Dublin 2, Ireland \\ email: brendan.goldsmith@dit.ie \\ and \\ K. Gong \\ School of Mathematical Sciences \\ Dublin Institute of Technology \\ Kevin Street, Dublin 8, Ireland \\ email:ketao.gong@mydit.ie
}

October 27, 2011

*2000 Mathematics Subject Classification. Primary: 20K10. Secondary: 20K21. Key words and phrases: Abelian groups, Hopfian groups, co-Hopfian groups. 


\begin{abstract}
The notions of Hopfian and co-Hopfian groups have been of interest for some time. In this present work we exploit some unpublished ideas of Corner to answer questions relating to such groups. In particular we extend an answer given by Corner to a problem of Beaumont and Pierce and show how the properties may be lifted from subgroups to the whole group in certain situations.
\end{abstract}

\title{
1 Introduction
}

The classes of groups which are today called Hopfian and co-Hopfian groups were first studied by Baer [1], under the names $Q$-group and $S$-group. In modern terminology we say that a group $G$ is Hopfian if every surjection $G \rightarrow G$ is an automorphism; it is said to be co-Hopfian if every injection $G \rightarrow G$ is an automorphism. Finite groups are, of course, the prototypes for both Hopfian and co-Hopfian groups. The existence of infinite co-Hopfian $p$-groups was first established by Crawley [4]. Hopfian and co-Hopfian groups have arisen recently in the study of algebraic entropy and its dual, adjoint entropy - see e.g. [5, 8]. Despite the seeming simplicity of their definitions, Hopfian and co-Hopfian groups are notoriously difficult to handle, for example, it is still not known whether the direct sum of two co-Hopfian groups which are not torsion-free, is co-Hopfian.

Our motivation for this work arose from some unpublished work of the late A.L.S. Corner, which we have adapted and extended to use in the context of Hopfian and co-Hopfian groups. In the first section we quickly review some standard results and consider the question of when subgroups inherit the Hopfian or co-Hopfian properties. We show, under a suitable simple condition, that the properties 'lift' from certain subgroups to the whole group; our argument is based on a result which may be of independent interest and utilizes arguments reminiscent of those used by Pierce [10] in his seminal work on homomorphism groups. In the final section we utilize an idea from an unpublished paper of A.L.S. Corner which answered a conjecture of Beaumont and Pierce [2], to exhibit, without assuming $(\mathrm{CH})$, mixed Hopfian and co-Hopfian groups with torsion subgroups of arbitrary cardinality $\lambda \leq 2^{\aleph_{0}}$. Moreover, the groups are the extension of a non-Hopfian (non-coHopfian) group by a non-Hopfian (non-co-Hopfian) group.

The word group shall normally mean an additively written Abelian group; the books [6] shall serve as a reference to ideas needed in Abelian group theory. We shall denote the set of primes by the symbol $\mathbb{P}$. 


\section{Lifting Hopficity (co-Hopficity) from Subgroups}

We begin by recording some well-known properties of Hopfian and co-Hopfian groups.

Definition 2.1 A group $G$ is said to be Hopfian if every surjection $G \rightarrow G$ is an automorphism; it is said to be co-Hopfian if every injection $G \rightarrow G$ is an automorphism.

It is easy to show that the Hopfian property for $G$ is equivalent to $G$ having no proper isomorphic factor group, while co-Hopficity is equivalent to having no proper isomorphic subgroup. The groups $\mathbb{Z}$ and $\mathbb{Z}\left(p^{\infty}\right)$ show that the notions are independent of each other.

The following simple proposition records some well-known and easily established facts about Hopficity and co-Hopficity:

Proposition 2.2 (i) A torsion-free group $G$ is co-Hopfian if, and only if it is divisible of finite rank.

(ii) A torsion-free group of finite rank is Hopfian.

(iii) Finitely generated groups are Hopfian and finitely co-generated groups are co-Hopfian.

(iv) A group $G$ with $\operatorname{End}(G) \cong \mathbb{Z}$ is Hopfian; thus arbitrarily large Hopfian groups exist.

(v) Reduced Hopfian (co-Hopfian) p-groups are semi-standard and so have cardinality at most $2^{\aleph_{0}}$.

(vi) A reduced countable Hopfian (co-Hopfian) p-group is finite.

The classes of Hopfian and co-Hopfian groups exhibit some weak closure properties which are well known:

Proposition 2.3 Let $0 \rightarrow H \rightarrow G \rightarrow K \rightarrow 0$ be an exact sequence.

(i)If $H, K$ are both Hopfian and if $H$ is left invariant by each surjection $\phi: G \rightarrow G$, then $G$ is Hopfian. In particular, extensions of Hopfian torsion groups by torsion-free Hopfian groups are again Hopfian.

(ii)If $H, K$ are both co-Hopfian and if $H$ is left invariant by each injection $\psi: G \rightarrow G$, then $G$ is co-Hopfian. In particular, extensions of co-Hopfian torsion groups by torsion-free co-Hopfian groups are again co-Hopfian.

Proof The proofs of the two statements are essentially dual, so we present only the proof of the Hopfian property. Let $\phi: G \rightarrow G$ be a surjection, then by assumption, $H \phi \leq H$ and so we get an induced map $\bar{\phi}: G / H \rightarrow G / H$ giving the following commutative diagram:

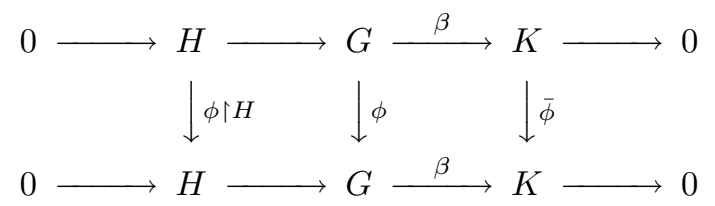


Since $\phi$ is onto, $\bar{\phi}$ is onto and so $K$, being Hopfian, gives that $\bar{\phi}$ is an automorphism. If we show that $\phi \uparrow H: H \rightarrow H$ is onto, then as $H$ is Hopfian, $\phi \uparrow H$ will also be an automorphism and the result will follow by an appeal to the "Five Lemma". However the fact that $\phi \uparrow H$ is onto follows immediately from the commutativity of the first square of the diagram above.

The following example, which provided the first examples of unbounded Hopfian and co-Hopfian p-groups, will be useful.

Example 2.4 If $B$ is a standard basic p-group and $G$ is a pure subgroup of the torsion-completion $\bar{B}$ of $B$ with $\operatorname{End}(G)=J_{p} 1_{G} \oplus E_{s}(G)$, where $E_{s}(G)$ is the ideal of small endomorphisms, then $G$ is both Hopfian and co-Hopfian.

Proof The details of this result are contained in Section 16 of Pierce's fundamental work [10]. The critical part of his argument is that a group with this type of endomorphism ring does exist; other proofs using variations of a realization theorem due to Corner [3] are possible. So assume such a group exists and suppose that $\psi=r+\theta$, where $r \in J_{p}$ and $\theta \in E_{s}(G)$, is a monic (epic) endomorphism of $G$, then using Lemma 16.1 in [10], we conclude that $r$ must be a $p$-adic unit. Moreover, Lemma 16.3 of [10] shows that if $B=\bigoplus_{n=1}^{\infty} B_{n}$, where each $B_{n}$ is a direct sum of cyclic groups of order $p^{n}$, then there are decompositions $G=B_{1} \oplus \cdots \oplus B_{m} \oplus H_{m}=B_{1} \oplus \cdots \oplus B_{m} \oplus H_{m} \psi$, where $H_{m}=\left\langle p^{m} G, B_{m+1}, \ldots\right\rangle$. Now, if $\psi$ is monic then $G \psi / H_{m} \psi \cong G / H_{m} \cong G / H_{m} \psi$ and so, since $G / H_{m}$ is finite, we have $G=G \psi$ and $\psi$ is an automorphism. If $\psi$ is epic, then $G /\left(H_{m}+\right.$ $\operatorname{Ker} \psi) \cong G / H_{m} \psi \cong G / H_{m}$ and again finiteness yields that $H_{m}+\operatorname{Ker} \psi=H_{m}$. Since $r$ is a unit, it follows that $H_{m}$ and $\operatorname{Ker} \psi$ are disjoint, whence $\operatorname{Ker} \psi=0$ and $\psi$ is again an automorphism.

If $G$ is a Hopfian (co-Hopfian) group then it is easy to see that subgroups of $G$ do not necessarily inherit this property: for example, if $G$ is an unbounded group which is both Hopfian and coHopfian as in Example 2.4, then a basic subgroup of $G$ is an unbounded direct sum of cyclic groups and hence is neither Hopfian nor co-Hopfian. However, we do have:

Proposition 2.5 If $G$ is Hopfian (co-Hopfian), then, for each natural number $n$, the subgroup $n G$ is Hopfian (co-Hopfian).

Proof If $\phi: n G \rightarrow n G$ is epic (monic), then it follows from the proof of Proposition 113.3 in [6], that there exists an epic (monic) $\psi: G \rightarrow G$ such that $\psi\lceil n G=\phi$. Since $G$ is Hopfian (co-Hopfian), $\psi$ must be an automorphism and hence its restriction to $n G$ is also an automorphism, i.e. $\phi$ is an automorphism.

The converse of Proposition 2.5 is not true in general: consider the $p$-group $G$ constructed by Pierce in [10, Theorem 16.4] which is both Hopfian and co-Hopfian - see Example 2.4 above. It follows 
from the last proposition that $p G$ is also both Hopfian and co-Hopfian. Now set $H=\bigoplus_{\aleph_{0}} \mathbb{Z}(p) \oplus G$; clearly $H$ is neither Hopfian nor co-Hopfian but $p H=p G$ has both properties.

We now show that, under a suitable restriction, a converse to Proposition 2.5 may be obtained. The key result is derived using arguments similar to those used by Pierce in [10] and may be of some independent interest.

First we make an ad hoc definition first used by Corner in unpublished work: an endomorphism $\epsilon$ of the group $G$ is said to be a q-map if $q(\epsilon-\alpha)=0$ for some automorphism $\alpha$ of $G$.

Theorem 2.6 If $G$ is a group which has no q-bounded pure subgroup of infinite rank and $q G$ is Hopfian (co-Hopfian), then $G$ is Hopfian (co-Hopfian).

Proof Let $\phi$ be an epic (monic) endomorphism of $G$. Then $\phi\lceil q G$ is an epic (monic) endomorphism of $q G$ and hence is an automorphism of $q G$. It follows from [6, Proposition 113.3] that there is an automorphism $\psi$ (say) of $G$ such that $\psi \uparrow q G=\phi \uparrow q G$. Consequently $q(\psi-\phi)=0$ and $\phi$ is a $q$-map of $G$. The result now follows immediately from Theorem 2.7 below.

Theorem 2.7 Suppose $G$ is a group which has no nonzero q-bounded pure subgroup for some integer $q=p_{1}^{k_{1}} p_{2}^{k_{2}} \ldots p_{t}^{k_{t}}$. Then if $\epsilon: G \rightarrow G$ is either monic or epic and a $q$-map, then $\epsilon$ is an automorphism of $G$.

Proof We remark at the outset that there is no loss in generality in assuming that $q \epsilon=q 1_{G}$ : since $\epsilon$ is a $q$-map, there is an automorphism $\alpha$ with $q \epsilon=q \alpha$, then simply replace $\epsilon$ by $\epsilon \alpha^{-1}$ and note that $\epsilon$ is epic (monic) if, and only if, $\epsilon \alpha^{-1}$ has the same property. We consider the three possibilities for $G$, i.e. $G$ is torsion-free, torsion or mixed. If $G$ is torsion-free, then $\epsilon=1_{G}$ and hence is an automorphism. Suppose then that $G$ is torsion and let $G_{i}(1 \leq i \leq t)$ denote the $p_{i}$-primary component of $G$. Then $G=\bigoplus_{i=1}^{t} G_{i} \oplus G_{0}$ for some complement $G_{0}$ having no $p_{i}$-primary component $(1 \leq i \leq t)$. Now each $G_{i}$ is left invariant by $\epsilon$. Moreover, the assumption that $G$ has no nonzero $q$-bounded pure subgroup means that the first $k_{i}$ Ulm invariants of each $G_{i}$ vanish. Then, it follows from Proposition 2.8 below that $\epsilon \uparrow G_{i}$ is an automorphism of $G_{i}$. Clearly $\epsilon$ acts as the direct sum of these restrictions and hence is an automorphism.

Finally, suppose that $G$ is mixed with torsion subgroup $T$. From the last paragraph it follows that $\epsilon\lceil T$ is an automorphism of $T$. Moreover the induced mapping $\bar{\epsilon}$ on $G / T$ is also an automorphism as noted above, since $G / T$ is torsion-free. It follows immediately from the Five Lemma that $\epsilon$ is an automorphism of $G$.

The final step in the proof of Theorem 2.7 is completed by the following more general result: 
Proposition 2.8 Let $G$ be a p-group such that the first $r$ Ulm invariants $f_{G}(0), f_{G}(1)$,

$f_{G}(2), \ldots, f_{G}(r-1)$ are finite and let $B=\bigoplus_{i=1}^{\infty} B_{i}$, where each $B_{i}$ is a direct sum of cyclic groups of order $p^{i}$, be a basic subgroup of $G$. If $\epsilon: G \rightarrow G$ is monic (respectively epic) such that $p^{r} \epsilon=p^{r} 1_{G}$, then $\epsilon$ is an automorphism of $G$.

Proof. Let $G=B_{1} \oplus \cdots \oplus B_{r} \oplus H_{r}$, where $H_{r}=\left\{p^{r} G, B_{r+1}, B_{r+2}, \ldots\right\}$; note that $B_{1} \oplus \cdots \oplus B_{r}$ is then finite.

(i) Claim that $\epsilon \uparrow H_{r}$ is monic.

Observe firstly that $\epsilon\left\lceil H_{r}[p]\right.$ is the identity map since $H_{r}[p] \leq p^{r} G$. Hence if $0 \neq x \in H_{r} \cap \operatorname{Ker} \epsilon$, there is a $k \geq 0$ such that $0 \neq p^{k} x \in H_{r}[p]$ and this would lead to the contradiction that $0=$ $p^{k}(x \epsilon)=\left(p^{k} x\right) \epsilon=p^{k} x \neq 0$.

(ii) Claim that $H_{r} \epsilon$ is pure in $G$.

It suffices to check this on the socle, so suppose that $x \in H_{r} \in \cap G[p] \cap p^{n} G$ for an arbitrary $n$. Then $x=y \epsilon$ for some $y \in H_{r}$. Since $\epsilon$ is monic on $H_{r}$, then $p x=0$ implies that $p y=0$ and so $y \in H_{r}[p]$. It follows from (i) above that $x=y \epsilon=y \in H_{r}[p]$. Thus $x \in H_{r} \cap p^{n} G=p^{n} H_{r}$ and so $x=p^{n} z$ for some $z \in H_{r}$. It now follows that $x=x \epsilon=p^{n} z \epsilon$ and so $x \in p^{n}\left(H_{r} \epsilon\right) \cap G[p]$ as required.

(iii) Claim that $H_{r}[p]=H_{r} \in[p]$.

It follows from (i) that the LHS of the above is contained in the RHS. So suppose that $z \in H_{r} \epsilon[p]$. Then $z=x \epsilon$ for some $x \in H_{r}$ and $0=p z=(p x) \epsilon$. Since $\epsilon$ is monic on $H_{r}$, then it is immediate that $x \in H_{r}[p]$, and then by (i) above, we have $z=x \epsilon=x \in H_{r}[p]$.

It follows from (ii) and (iii) that $H_{r}$ and $H_{r} \epsilon$ are pure subgroups of $G$ having equal socles. Since $H_{r}$ is a summand, it follows from a result of Irwin and Walker [9, Theorem 16], that $H_{r} \epsilon$ is also a summand and that it has a common complement to $H_{r}$. Thus $G=H_{r} \oplus X=H_{r} \epsilon \oplus X$ where $X \cong B_{1} \oplus \cdots \oplus B_{r} ;$ as observed above, $X$ is finite.

If $\epsilon$ is monic, it induces an isomorphism $G / H_{r} \cong G \epsilon / H_{r} \epsilon$. Moreover $G / H_{r} \cong G / H_{r} \epsilon$, and so both of these groups are then finite. It follows immediately that $G=G \epsilon$ and $\epsilon$ is an automorphism, as required. If $\epsilon$ is epic, then the mapping $x \mapsto x \epsilon+H_{r} \epsilon$ has kernel $H_{r}+$ Ker $\epsilon$ and so there are isomorphisms $G / H_{r}+\operatorname{Ker} \epsilon \cong G / H_{r} \epsilon \cong G / H_{r}$. Again all the groups are finite and so $H_{r}+\operatorname{Ker} \epsilon=$ $H_{r}$. However if $x \in \operatorname{Ker} \epsilon[p]$, then $x \in H_{r}[p]$ and so from (i) above, $0=x \epsilon=x$. Thus Ker $\epsilon=0$ and $\epsilon$ is again an automorphism.

\section{$3 \quad$ Mixed groups}

We now switch our attention to mixed Abelian groups. Our approach in this section is heavily influenced by an unpublished paper of Corner answering a conjecture of Beaumont and Pierce - 
this is [U16] in [7]. We proceed via a series of steps starting with an arbitrary unbounded semistandard (not necessarily separable) reduced $p$-group $T$. Firstly we determine the structure of the quotient $A / T$, where $A$ is the cotorsion-completion of $T$ and then we construct a subgroup $H$ of the group of $p$-adic integers $J_{p}$ with $\operatorname{End}(H)=\mathbb{Z}_{p}$, the integers localized at the prime $p$. Finally we use $A$ and $H$ to construct, via a suitable pullback, a mixed group $G$ which will be both Hopfian and co-Hopfian.

(1) So suppose that $T$ is an arbitrary unbounded semi-standard (not necessarily separable) reduced $p$-group and let $A=\operatorname{Ext}(\mathbb{Q} / \mathbb{Z}, T)$. Then $A$ is the cotorsion-completion, $T^{\bullet}$, of $T$ and $A / T \cong$ $\operatorname{Ext}(\mathbb{Q}, T)$ is torsion-free divisible. We claim $A / T \cong \bigoplus_{2^{N_{0}}} \mathbb{Q}$.

To establish the claim note that if $B$ is a basic subgroup of $T$, then $B$ is pure and dense in $T$ and so there is an epimorphism $\operatorname{Ext}(\mathbb{Q}, B) \rightarrow \operatorname{Ext}(\mathbb{Q}, T)$. Moreover, $B$ is, by Szele's Theorem, an epimorphic image of $T$ and so there is an epimorphism $\operatorname{Ext}(\mathbb{Q}, T) \rightarrow \operatorname{Ext}(\mathbb{Q}, B)$. Since $\mathbb{Q}$ is torsion-free and divisible, both $\operatorname{Ext}(\mathbb{Q}, B)$ and $\operatorname{Ext}(\mathbb{Q}, T)$ are $\mathbb{Q}$-vector spaces and the existence of the above epimorphisms ensures that they are of the same dimension. Hence $\operatorname{Ext}(\mathbb{Q}, B) \cong$ $\operatorname{Ext}(\mathbb{Q}, T)$; furthermore, the algebraic compactness of $\hat{B}$ implies that $\operatorname{Ext}(\mathbb{Q}, B) \cong \operatorname{Hom}(\mathbb{Q}, \hat{B} / B)$. We remark that the exact structure of the quotient $\hat{B} / B$ may be computed but it is not essential for our purposes here: it suffices to note that as it is divisible, it has the form $\hat{B} / B \cong \bigoplus_{\lambda} \mathbb{Q} \oplus$ $\bigoplus_{\kappa} \mathbb{Z}\left(p^{\infty}\right)$, where $\max \{\lambda, \kappa\}=2^{\aleph_{0}}$. Thus $\operatorname{Hom}(\mathbb{Q}, \hat{B} / B) \cong \operatorname{Hom}\left(\mathbb{Q}, \bigoplus_{\lambda} \mathbb{Q}\right) \oplus \operatorname{Hom}\left(\mathbb{Q}, \bigoplus_{\kappa} \mathbb{Z}\left(p^{\infty}\right)\right)$. The first term is easily seen to be isomorphic to $\bigoplus_{\lambda} \mathbb{Q}$. Now $\operatorname{Hom}\left(\mathbb{Q}, \bigoplus_{\kappa} \mathbb{Z}\left(p^{\infty}\right)\right)$ is torsion-free divisible since so is $\mathbb{Q}$, and it has cardinality $\leq\left(2^{\aleph_{0}}\right)^{\aleph_{0}}=2^{\aleph_{0}}$ since $\kappa \leq 2^{\aleph_{0}}$. However, $\mathbb{Z}\left(p^{\infty}\right)$ is an epimorphic image of $\mathbb{Q}$ and so $\operatorname{Hom}\left(\mathbb{Q}, \bigoplus_{\kappa} \mathbb{Z}\left(p^{\infty}\right)\right)$ also contains a subgroup isomorphic to $\operatorname{Hom}\left(\mathbb{Z}\left(p^{\infty}\right), \bigoplus_{\kappa} \mathbb{Z}\left(p^{\infty}\right)\right)$; this latter is isomorphic to $\widehat{\bigoplus}_{\kappa} J_{p}$ and hence has cardinality $\geq 2^{\aleph_{0}}$. Thus $\operatorname{Hom}\left(\mathbb{Q}, \bigoplus_{\kappa} \mathbb{Z}\left(p^{\infty}\right)\right) \cong \bigoplus_{2^{\aleph_{0}}} \mathbb{Q}$ and in any event we have that $\operatorname{Hom}(\mathbb{Q}, \hat{B} / B) \cong \bigoplus_{2^{\aleph_{0}}} \mathbb{Q}$ and so $A / T \cong \underset{2^{\aleph_{0}}}{\bigoplus} \mathbb{Q}$, as claimed.

Note that if $\phi: A \rightarrow T$ is any homomorphism, then the image $A \phi$ is both cotorsion and torsion, and hence is bounded [6, Corollary 54.4].

(2) Let $H$ be a maximal pure subgroup of $J_{p}$ containing $\mathbb{Z}_{p}$, then $H$ has cardinality $2^{\aleph_{0}}$ and $J_{p} / H \cong \mathbb{Q}$. Since every endomorphism of $H$ extends to an endomorphism of $J_{p}$, it must be multiplication by a $p$-adic integer. Moreover, this multiplication must induce an endomorphism on the quotient $J_{p} / H \cong \mathbb{Q}$, and so it must be both a $p$-adic integer and a rational integer i.e. it is in $\mathbb{Z}_{p}$. Conversely since $J_{p}$ is $q$-divisible for all primes $q \neq p$, any multiplication by an element of $\mathbb{Z}_{p}$ is an endomorphism of $H$.

Thus we have a pure subgroup $H$ of the group of $p$-adic integers $J_{p}$ such that $H$ contains the subgroup $\mathbb{Z}_{p}$ of integers localized at $p$ and $\operatorname{End}(H)=\mathbb{Z}_{p}$. Moreover, as $H$ has rank $2^{\aleph_{0}}$, we have 
that $H / \mathbb{Z}_{p}$ is torsion-free divisible of rank $2^{\aleph_{0}}$.

(3) We now use the groups $A$ and $H$ constructed above to construct a mixed group $G$.

The groups $A / T$ and $H / \mathbb{Z}_{p}$ are isomorphic, fixing such an isomorphism we form the pullback of $A$ and $H$ with kernels $T$ and $\mathbb{Z}_{p}$. The resulting group $G$ is a subgroup of the direct sum $A \oplus H$ and satisfies

$$
G / T \cong H, G / \mathbb{Z}_{p} \cong A
$$

Since $G / T$ is torsion-free, $T$ is the torsion subgroup of $G$. Note that $G / T$ is reduced in this case.

We claim that the group $G$ so constructed is both co-Hopfian and Hopfian.

To see this, suppose that $\epsilon: G \rightarrow G$ is any monomorphism (respectively epimorphism) of $G$; we shall show that $\epsilon$ is an automorphism of $G$. Now, $\epsilon$ induces a mapping $\bar{\epsilon}$ of $G / T \cong H$ and so $\bar{\epsilon}$ is multiplication by a rational $n / m$ where $n, m$ are coprime and $p \nmid m$. Thus, $m \epsilon-n 1_{G}$ induces the zero map on $G / T$ and so $G\left(m \epsilon-n 1_{G}\right) \leq T$, in particular $\mathbb{Z}_{p}\left(m \epsilon-n 1_{G}\right) \leq T$. However, every homomorphic image of $\mathbb{Z}_{p}$ in $T$ is cyclic, and so bounded, so there exists an integer $r \geq 0$ such that $p^{r} \mathbb{Z}_{p}\left(m \epsilon-n 1_{G}\right)=0$. Thus, the endomorphism $p^{r}\left(m \epsilon-n 1_{G}\right)$ of $G$ annihilates $\mathbb{Z}_{p}$ and so passes to the quotient inducing a map: $G / \mathbb{Z}_{p} \cong A \rightarrow T$; as noted above the choice of $A$ means this image is also bounded. So replacing $r$ by a larger integer if necessary, we may suppose that this image is zero i.e. $p^{r}\left(m \epsilon-n 1_{G}\right)=0$.

We consider firstly the case where $\epsilon$ is monic. Since $T$ is unbounded there is a cyclic summand $\langle x\rangle$ of $T$ of order greater than $p^{r}$; say $O(x)=r+s$. Thus, $p^{r} m\left(p^{s-1} x\right) \neq 0$ and so $p^{r} n\left(p^{s-1} x\right)=$ $p^{r} m\left(p^{s-1} x\right) \epsilon \neq 0$ as $\epsilon$ is monic. Hence, $p \nmid n$ and $n / m$ is a unit in $\mathbb{Z}_{p}$.

If $\epsilon$ is epic, then note that since $T$ is reduced and unbounded, $p^{r} T>p^{r+1} T$. However, as $\epsilon$ is onto and $p \nmid m,\left(p^{r} T\right) m \epsilon=p^{r} T$, but on the other hand $\left(p^{r} T\right) m \epsilon=p^{r} n T$ and we deduce immediately that $p \nmid n$ and $n / m$ is a unit in $\mathbb{Z}_{p}$.

Thus, in either case, the endomorphism induced on $G / T$ by $\epsilon$, is in fact an automorphism. To prove that $\epsilon$ is an automorphism of $G$, it is enough, by the Five Lemma, to prove that $\epsilon$ induces an automorphism of $T$. Moreover, as multiplication by $n / m$ effects an automorphism of $T, n / m(\epsilon\lceil T)$ is a monomorphism (respectively an epimorphism) $T \rightarrow T$ and it is enough to prove it is an automorphism of $T$. In other words we may restrict attention to the case $n=m=1$.

Then $\epsilon: T \rightarrow T$ is a monomorphism (respectively an epimorphism) such that $p^{r}\left(\epsilon-1_{T}\right)=0$ for some $r \geq 1$. We show that $\epsilon$ is an automorphism of $T$. (When $T$ is the torsion-completion of a standard basic group, it is possible to give a fairly direct element-wise proof of this fact. To the best of our knowledge, this was first done for monomorphisms by A.L.S. Corner in 1962 in an unpublished paper ([U16] in [7]) answering a conjecture of Beaumont and Pierce - see the conjecture before Example 1, p218 in [2].) This, however, is immediate from Theorem 2.7 above, 
since we have assumed that $T$ is semi-standard.

Summarizing the above, we have established:

Theorem 3.1 If $T$ is an arbitrary semi-standard unbounded reduced p-group, then there is a mixed Abelian group $G$, which is both Hopfian and co-Hopfian, and which satisfies

(i) $T$ is the torsion subgroup of $G$

(ii) the quotient $G / T$ is isomorphic to a pure subgroup $H$, of cardinality $2^{\aleph_{0}}$, of the group of p-adic integers $J_{p}$ (and, in particular, is reduced).

Our first corollary answers negatively a conjecture of Beaumont and Pierce [2] mentioned above. Note that in their terminology an $I$-group is precisely a group which is not co-Hopfian.

Corollary 3.2 For any infinite cardinal $\lambda \leq 2^{\aleph_{0}}$, there exists a mixed Abelian group $G$ with torsion subgroup $T$ of cardinality $\lambda$, such that $G / T$ is reduced and $G$ is co-Hopfian (and hence not an I-group) and Hopfian.

Proof Let $B$ be a semi-standard $p$-group so that the quotient $D=\bar{B} / B \cong \bigoplus_{2^{\aleph_{0}}} \mathbb{Z}\left(p^{\infty}\right)$. Let $T$ be the pre-image in $\bar{B}$ of a subgroup $D_{1}$ of $D$ such that $D_{1} \cong \bigoplus_{\lambda} \mathbb{Z}\left(p^{\infty}\right)$. Then $T$ is a semistandard separable $p$-group of cardinality $\lambda$. So, by Theorem 3.1, there is a co-Hopfian group $G$ with $t(G)=T$ and $G / T$ is a reduced subgroup of $J_{p}$.

The mixed groups we have constructed, in contrast to Proposition 2.3, can have the property that they are the extension of a non-co-Hopfian fully invariant subgroup by a non-co-Hopfian group, and yet are co-Hopfian: simply choose $T$ to be a standard basic group which is clearly not coHopfian and observe that a pure subgroup of $J_{p}$ with endomorphism ring $\mathbb{Z}_{p}$, is not co-Hopfian since multiplication by $p$ is a monomorphism which is not an automorphism.

We conclude this brief discussion of Hopfian and co-Hopfian groups by raising a number of problems; we believe that solutions to these problems would give a great deal of insight into the structure of such groups. Our first problem comes from the observation that reduced $p$-groups constructed to date seem to possess both properties or neither.

Problem 1 Find a reduced $p$-group which is Hopfian but not co-Hopfian and vice versa.

We have noted above that countable Hopfian (co-Hopfian) $p$-groups are finite and that there exist Hopfian and co-Hopfian groups of cardinality $2^{\aleph_{0}}$. Thus we pose:

Problem 2 Assuming the negation of the Continuum Hypothesis, do there exist Hopfian (coHopfian) $p$-groups of cardinality $\kappa$ for $\aleph_{0}<\kappa<2^{\aleph_{0}}$ ? 


\section{References}

[1] R. Baer, Groups without Proper Isomorphic Quotient Groups, Bull. Amer. Math. Soc. 50 (1944) $267-278$.

[2] R.A. Beaumont and R.S. Pierce, Partly Transitive Modules with Proper Isomorphic Submodules, Trans. Amer. Math. Soc. 91 (1959) 209 - 219.

[3] A. L. S. Corner, On endomorphism rings of primary Abelian groups, Quart. J. Math. Oxford 20 (1969) $277-296$.

[4] P. Crawley, An infinite primary group without proper isomorphic subgroups, Bull. Amer. Math. Soc. 68 (1962) 463 - 467.

[5] D. Dikranian, B. Goldsmith, L. Salce and P. Zanardo, Algebraic entropy for Abelian groups, Trans Amer. Math. Soc. 361 (2009) 3401 - 3434.

[6] L. Fuchs, Infinite Abelian Groups, Vol. I and II, Academic Press, 1970 and 1973.

[7] B. Goldsmith Anthony Leonard Southern Corner 1934-2006, in Models, Modules and Abelian Groups, (editors R. Göbel and B. Goldsmith) Walter de Gruyter, Berlin (2008) 1 - 7.

[8] B. Goldsmith and K. Gong, On adjoint entropy of Abelian groups, to appear in Comm. Algebra.

[9] J.M. Irwin and E.A. Walker, On N-High Subgroups of Abelian Groups, Pacific J. Math. 11 (1960) $1363-1374$.

[10] R.S. Pierce, Homomorphisms of primary Abelian groups, in Topics in Abelian Groups, Scott Foresman (1963) 215 - 310. 